\title{
Spatially Resolved Study of Inter-Cusp Transport and Containment of Primary Electrons
}

\author{
Aimee A. Hubble ${ }^{1}$ and John E. Foster ${ }^{2}$ \\ University of Michigan, Ann Arbor, MI, 48105
}

\begin{abstract}
Electron current density profiles were obtained in the region above two magnetic cusps in a $20 \mathrm{~cm}$ partial conic ring cusp ion thruster discharge chamber. The density profiles were obtained by use of a translatable Langmuir probe and an automated motion stage and data collection system. These high-resolution density profiles allowed for the study of electron collection mechanics in the absence of gas flow and plasma production, as well as at varying levels of gas flow. This is the second stage in a series of experiments aimed at better understanding collection physics at the magnetic cusps during discharge chamber operation. The current density maps allow for particle transport through the cusps to be visualized, and see how discharge chamber geometry plays a role in collection. Transmission coefficients and loss widths as a function of probe height above the anode were calculated from the current density data, and compared at a variety of discharge currents. In addition, these current density maps were compared to those obtained using a planar line cusp source geometry to study how source geometry and magnet cusp spacing affects collection.
\end{abstract}

\section{Introduction}

$\mathrm{T}$ HE physics of losses at magnetic cusps remains a fundamental yet not fully understood problem ${ }^{1-7}$; one that is crucial to accurately assessing the effective loss area at the wall in multipole ion sources. Effective loss area determines discharge ionization performance, efficiency, and stability, ${ }^{3,8,9}$, and is used in the modeling and design of ion source multipole discharge chambers. The ultimate goal of this work is to determine an effective loss area that can be used to quantify plasma losses to the wall in multipole discharge chambers.

A ring-cusp discharge chamber features a number of magnet rings of alternating polarity. This creates a magnetic field geometry in which particles are constrained along the strong field lines near the anode and are largely lost to the narrow regions defined by the magnetic cusps formed at the poles of the magnet rings as opposed to being lost uniformly over the entire anode surface. The path length of ionizing primary electrons is thus increased, and the electrons become more likely to undergo an ionizing collision before they are finally lost to the wall ${ }^{2,9-16}$.

The improvements to plasma containment from magnetic cusps have been extensively studied ${ }^{5,17-20}$, and improvements in permanent magnet configurations have produced the ring-cusp geometry used in modern thrusters $^{21,22}$. The density improvements observed in such configurations are the result of increased confinement of ionizing primary electrons ${ }^{16,23}$, as well as indirect enhancements to ion confinement from ambipolar diffusion effects $^{24}$. Past studies have focused on the loss width of the cusp at the discharge chamber wall. This width has been determined by Hershkowitz ${ }^{18}$ and others ${ }^{1,4,6}$ to be proportional to the hybrid gyroradius: $r_{h}=\sqrt{r_{e} \cdot r_{i}}$, for lowbeta plasmas. Recent work has shown, however, that the loss width alone does not give a complete accounting of losses to the wall. These studies ${ }^{25,26}$ have shown considerable attenuation in particle flux between the mouth of the cusp and the anode wall. This suggests that loss width coupled with a transmission coefficient may give a more accurate accounting of losses for a particular magnetic circuit.

Ultimately, the knowledge of this effective loss area can be used to design more efficient discharge chambers. This knowledge will provide for a fundamental understanding of the collection and trapping of ionizing primary electrons and plasma electrons at and between magnetic cusps. From any magnetic circuit, particle losses can be determined which will allow for prediction of discharge performance. Ultimately, the findings of this work will be incorporated into an energy and particle balance model ${ }^{27,28}$.

\footnotetext{
${ }^{1}$ Graduate Student, Nuclear Engineering and Radiological Sciences, 2355 Bonisteel Blvd, Student Member.

${ }^{2}$ Associate Professor, Nuclear Engineering and Radiological Sciences, 2355 Bonisteel Blvd, Full Member.
} 


\section{Experimental Setup}

Two experiments are described in this paper. In the first, a simple, planar line cusp geometry is investigated to determine the effect of filament spatial location and magnetic row symmetry on collection. In the second experiment, electron collection is assessed in a $20-\mathrm{cm}$ conical discharge chamber to represent a more thruster-like magnetic configuration. In both cases Langmuir probe current is measured above the magnetic cusps in a plane perpendicular to the magnet surface. This gives a 2D high-resolution current density map that allows for visualization of particle transport to the cusps.

\section{A. Planar Line Cusp Source}

Three permanent magnet line cusps were arranged on a 7.5 by $5 \mathrm{~cm}$ steel plate with mica backing, shown in Figure 1. The mica was intended to prevent collection on the back side of the plate, since it is the collection at the cusps that is of interest in this experiment. A single tungsten filament fashioned from $0.5 \mathrm{~mm}$ wire was placed 10 $\mathrm{cm}$ above the plate, and was coated in R500 coating to improve emission. The plate anode was biased $63.4 \mathrm{~V}$ positively relative to the filament and the entire setup was placed inside a vacuum chamber. The experiment was operated at a base pressure of $10^{-5}$ Torr. At this pressure it is expected that the current collected at the plate will be comprised largely of primary electrons. This is consistent with prior calculations of the probability of a primary electron undergoing an ionizing collision:

$$
P=1-\exp (-\sigma n L)
$$

where $\mathrm{P}$ is the ionization probability, $\sigma$ is the ionization cross section, $\mathrm{n}$ is the neutral number density, and $\mathrm{L}$ is the total path length of the primary electrons. Using a neutral density of $3^{*} 10^{17} \mathrm{~m}^{-3}$ (equivalent to $\mathrm{N}_{2}$ at room temperature and $1 \mathrm{e}-5$ Torr), an ionization cross section of $10^{-20}$ $\mathrm{m}^{2}$, and assuming a path length of $0.25 \mathrm{~m}$, a probability less than $0.1 \%$ is obtained. Thus we assume ionization is negligible, and that the probe current measured is due almost exclusively to primary electrons.

The experimental setup allowed for the filament position to be adjusted. The spacing between magnet rows could also be changed. This allowed for the investigation of the effect of these factors on collection at the magnet surface as well as cusp profile shape. A sample configuration of the planar line cusp source is shown in Figure 1. A complete experimental setup schematic is shown in Figure 2.

\section{B. 20-cm Conical Discharge Chamber}

The conical discharge chamber, depicted in Figure 3 in its experimental configuration, features four samarium-cobalt permanent magnet rings. It is $20 \mathrm{~cm}$ in diameter at the exit plane and $10 \mathrm{~cm}$

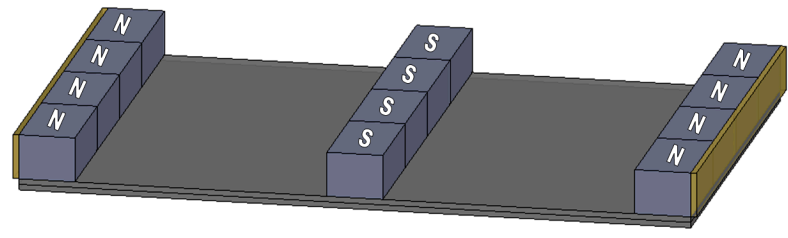

Figure 1: Planar Line Cusp Source.

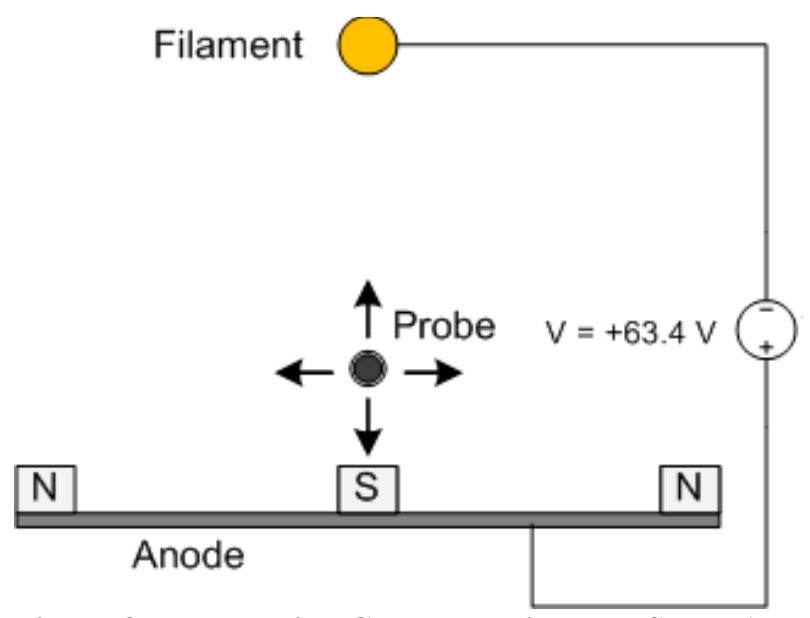

Figure 2: Planar Line Cusp Experimental Setup (not to scale). long. The mild steel discharge chamber body and the magnet rings form the thruster anode. Two tungsten filaments similar to the one used in the line cusp experiment were used as the thruster cathode. The filaments were also coated in R500 coating and were electrically isolated from the anode using ceramic standoffs. A stainless steel grid was mounted at the exit plane using ceramic standoffs. For these experiments, the anode was biased $63.4 \mathrm{~V}$ positively relative to the filament and the grid was biased $30 \mathrm{~V}$ below anode potential. The entire setup was placed in a vacuum chamber. This experiment was also operated at a base pressure of $10^{-5}$ Torr, at which pressure it is expected that the current collected at the plate and by the probe in the cusp region will be largely due to primary electrons. Ionization probability was again determined using Equation 1. The path length in this setup is given $b^{27}$ : 


$$
L=v_{p} * \tau_{p}=\frac{V}{A_{p}}
$$

where $\mathrm{V}$ is the volume of the chamber and $\mathrm{A}_{\mathrm{p}}$ is the loss area for primary electrons, calculated to be twice the primary Larmor radius at the magnet surface multiplied by the inner circumference of all four magnet rings. An effective path length of 4.7 $\mathrm{m}$ is thus obtained. The probability of a primary electron encountering an ionizing collision in that length of travel is calculated to be about $1.4 \%$, sufficiently low that ionization may be neglected.

The chamber was also operated in the case of argon gas flow, at pressures of $10^{-5}, 6.5^{*} 10^{-5}$, and $1.5^{*} 10^{-}$

${ }^{4}$ Torr, corresponding to ionization probabilities of about $1.4 \%, 20 \%$, and $40 \%$. This allowed for the study of collection as a function of ionization probability to ascertain the sensitivity of collection to the presence of plasma. Probe interrogations were carried out in the cusp region of two of the four magnet rings.

\section{Diagnostics}

A Langmuir probe with a planar collection surface was used to measure current density profiles across the magnet cusps in both experiments. The planar probe had a $1 \mathrm{~mm}^{2}$ tantalum collection surface and was mounted on a macor extension arm. The extension arm was affixed to a three-axis motion control system. The current collected by the probe is taken to be proportional to the current flowing along the cusp at that point. Probe voltage was supplied by a DC power supply and an electrometer was used to measure the probe current. Cusp probe currents were typically on the order of 10 to $100 \mathrm{nA}$. Uncertainty in probe measurements was on the order of nA.

\section{Filament}

\section{Planar Line Cusp Source}

Measurements were made with a resolution of $0.2 \pm$ $0.01 \mathrm{~mm}$ parallel to the magnet surface over a $95 \mathrm{~mm}$ interrogation track. The measurements are repeated at increments of $2 \mathrm{~mm}$ up to $20 \mathrm{~mm}$ above the magnet surface to produce a high-resolution current density map of the cusp region. The probe effectively traced out a plane perpendicular to the anode surface, allowing for the visualization of primary electron transport through the cusp region over all three line cusps. The area traced by the probe is depicted in Figure 4.

\section{20-cm Conic Discharge Chamber}

Measurements were made with a resolution of $0.2 \pm$ $0.01 \mathrm{~mm}$ parallel to the magnet surface over a $50 \mathrm{~mm}$ interrogation track, and were repeated at increments of 5 $\mathrm{mm}$ up to $35 \mathrm{~mm}$ above the magnet surface. The probe swept over two of the four magnet rings, allowing for visualization of primary electron transport through the cusp region of these two magnet rings. The area traced by the probe is depicted in Figure 5 .

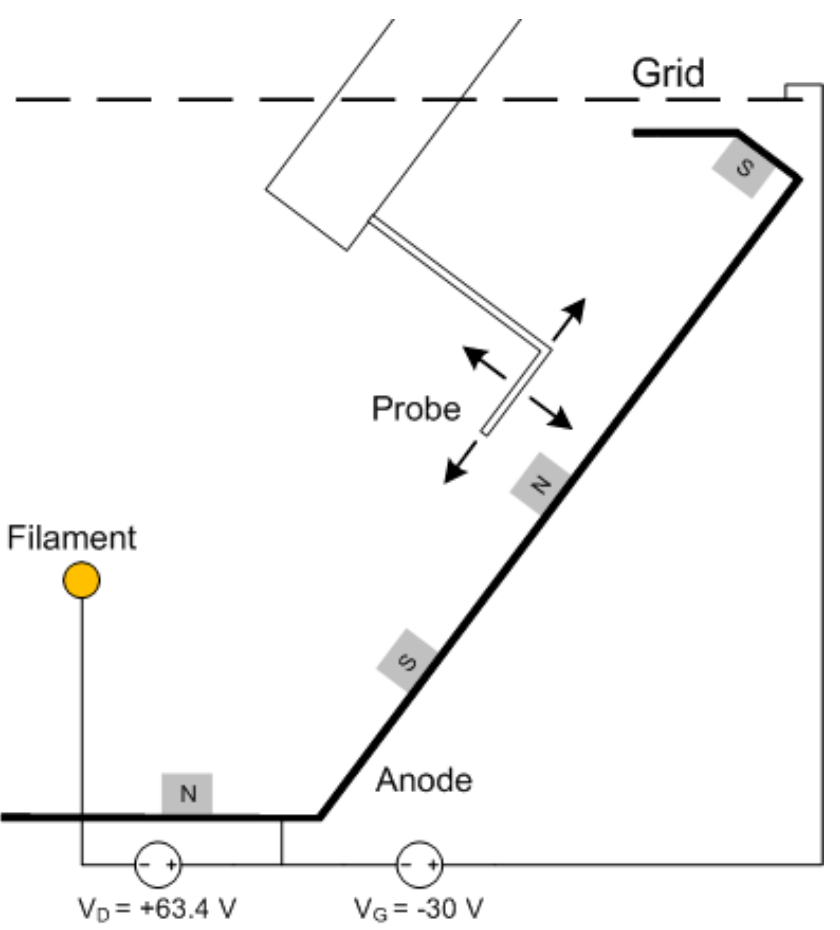

Figure 3: Experimental setup depicting cross-section of 20-cm conical discharge chamber with translatable Langmuir probe. 
A fixed Langmuir probe was mounted near the center of the discharge chamber to take measurements in the field-free region using an automated probe data acquisition system with a current resolution of $0.1 \mu \mathrm{A}$.

\section{Results and Discussion}

\section{A. Planar Line Cusp Source}

\section{Effect of filament placement on collection}

Five tests were conducted using the planar line cusp source, which studied filament placement and inter-cusp spacing and its effects on collection and cusp profile shape. The case of equal intermagnet row spacing was investigated first, as depicted in Figure 1. In this layout, the sensitivity of electron collection to filament placement was investigated at three locations: the center of the plate (i.e. directly above the central line cusp), 0.5 inches off-center, and 1.5 inches off-center (i.e. at the edge of the plate). The geometries investigated are represented schematically in Figure 6.

Measurements with the translatable probe were made as described in Section 2.C.1, and raw probe current is plotted as a function of position relative to the anode plate as indicated in Figure 7. As indicated, the majority of the current appears to collect at the middle magnet region. It is evident that as the filament is moved further from the center of the plate, the plate collects much less current in the cusp region as indicated by the drop off in probe current in the cusp region and also by the drop in electron emission current (from 10 $\mathrm{mA}$ to $6.5 \mathrm{~mA}$ ) at fixed voltage. At distances farther away, the magnetized region that the electrons must traverse is larger and in absence of significant neutral collision, mean diffusion from the cathode to the plate is reduced. In other words, at greater distances the electrons are more likely to encounter closed field loops, making access to the plate more difficult in the absence of an appreciable neutral electron collision frequency. At closer distances, electrons can actually be launched along field lines that terminate at the poles. It does not appear, however, that there is any significant asymmetry in current profile shape as the filament is moved offcenter.

Cusp profile width and transmission were determined for the profile measured over the central cusp. Profile width is taken to be the Full Width Half Maximum (FWHM) of the current profile measured by the probe at a particular height above the magnet surface. In this manner one can plot cusp width as a function of height over the magnet. This width is compared with twice the primary electron Larmor radius for each of the three cases investigated. The primary electron Larmor radius, $r_{p}$, was determined from Hall probe measurements of magnetic field as a function of height above the magnet and assuming primary electron energy was $63.4 \mathrm{eV}$; the discharge voltage. This is plotted in Figure 8.

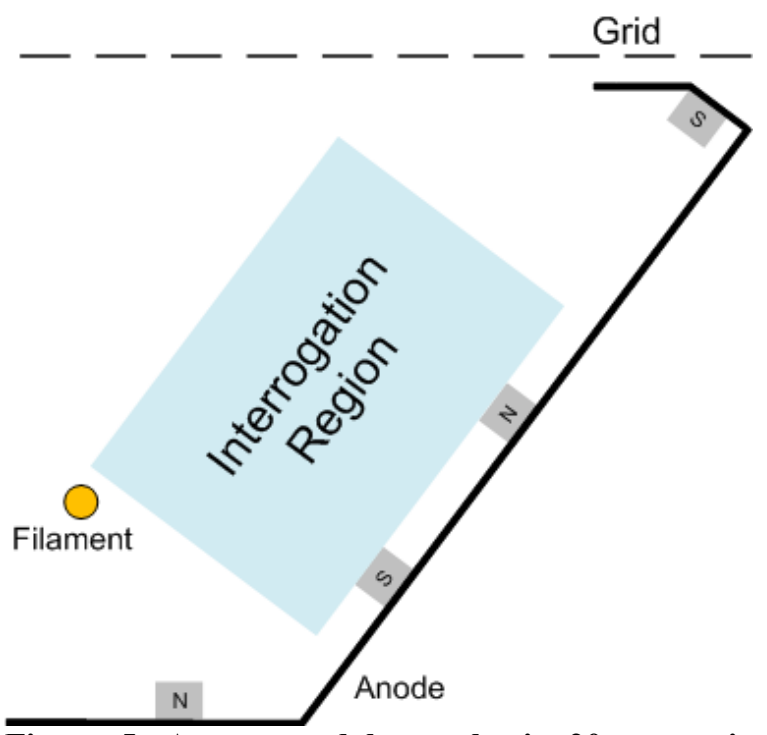

Figure 5: Area traced by probe in $20-\mathrm{cm}$ conic discharge chamber experiment.

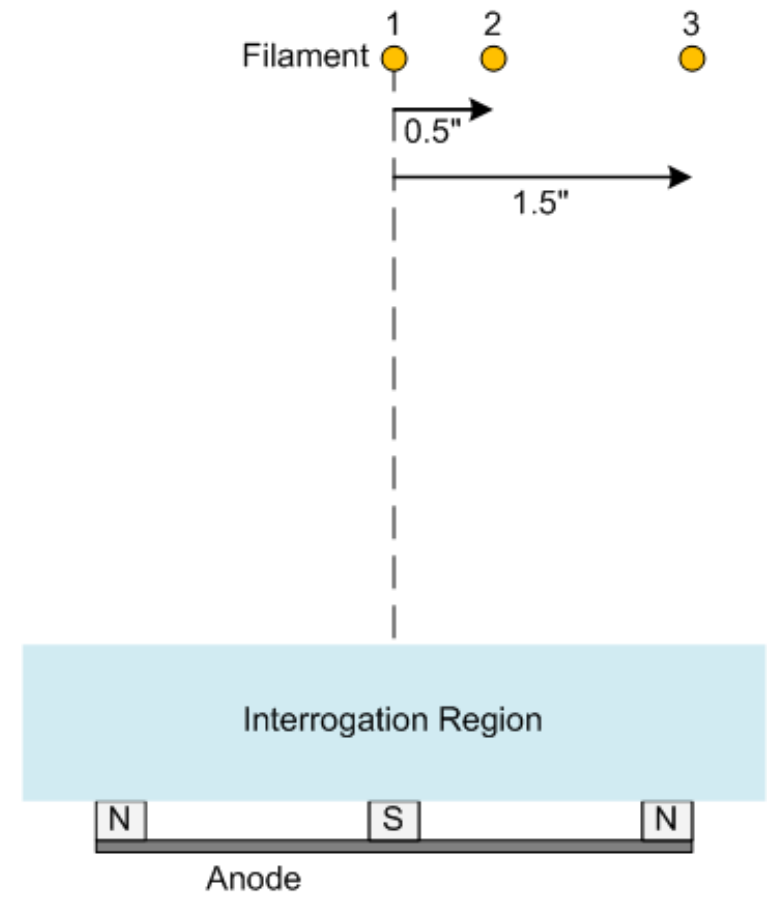

Figure 6: Filament placement for the first three studies. 


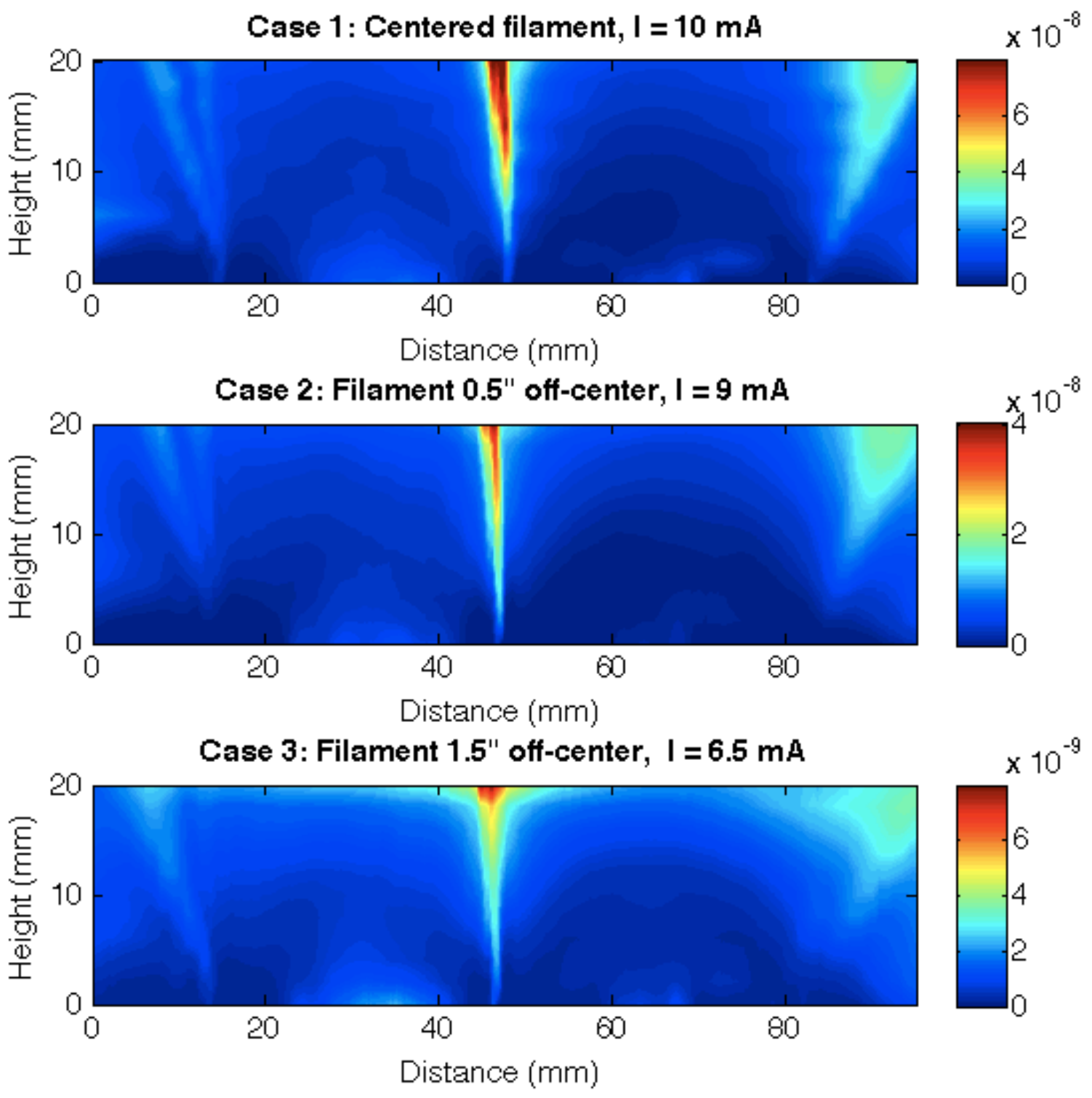

Figure 7: Raw probe current (A) as a function of position relative to the anode plate. Cases correspond with the geometries depicted in Figure 6. Note the difference in scale between the three plots.

Integrating the current measured by the probe at a particular height above the magnet surface across the width of the cusp gives a measure of total current incident on the anode as a function of height. Transmission is determined to be the ratio of total current at the anode to that at the cusp of the mouth, taken to be at $20 \mathrm{~mm}$ in the case of these measurements. Transmission is plotted for each of the three cases investigated in Figure 9. It is clear that filament placement appears to have no appreciable bearing on uncorrected cusp collection profiles or collected current magnitude, however collection profiles are artificially enlarged due to the finite size of the probe and therefore must be deconvoluted to get the true current profile.

\section{Effect of inter-cusp spacing on collection}

The positions of the line cusps were varied to affect the symmetry of the multi-dipole field along the center magnet row. Three cases were investigated, including the symmetric case shown in Figure 1 . The three configurations and corresponding magnetic field vector plots (from a commercial magnetostatic solver) are shown schematically in Figure 10, and raw probe current is plotted as a function of position relative to the anode plate in Figure 11. The filament position, which was located directly above the center of the plate, was not varied during 


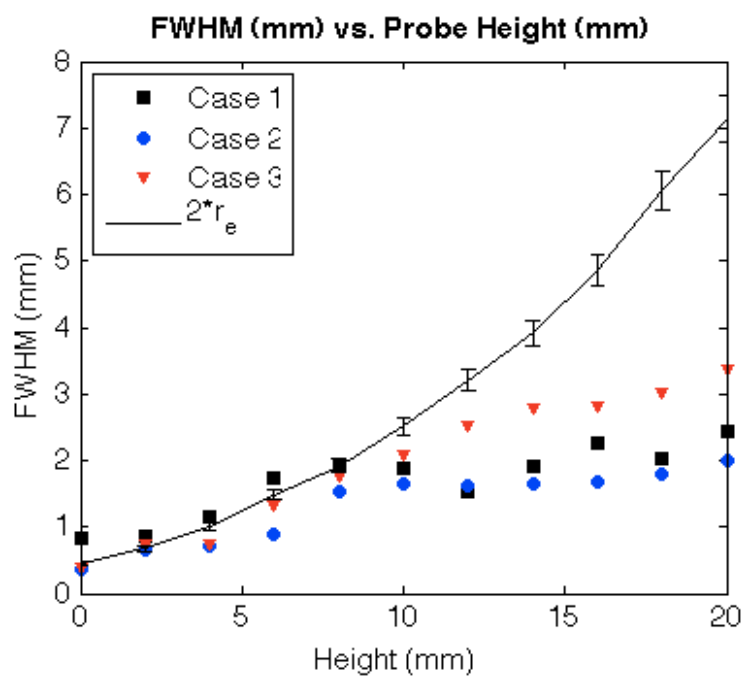

Figure 8: FWHM (mm) as a function of probe height above the magnet surface $(\mathrm{mm})$. These widths are compared with twice the primary electron Larmor radius (mm).

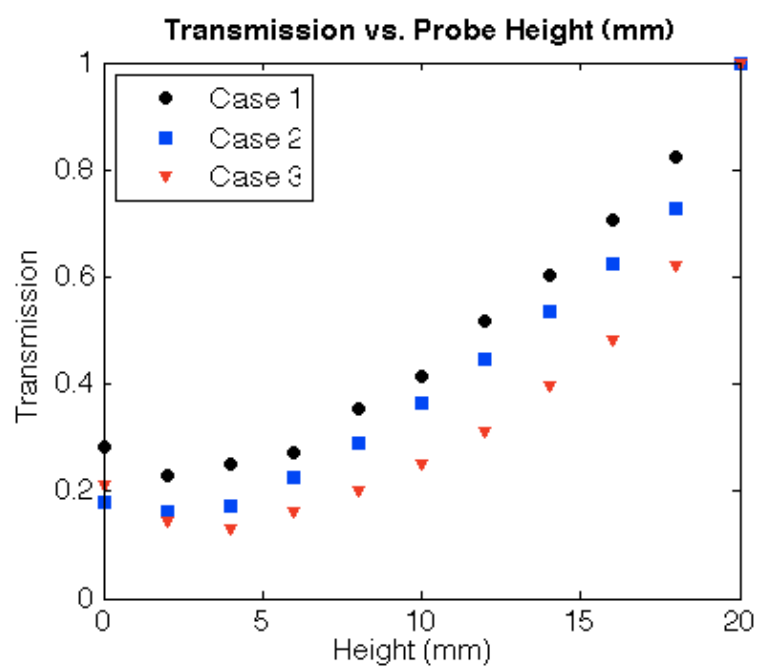

Figure 9: Transmission as a function of probe height above the magnet surface $(\mathrm{mm})$.

these tests. It is apparent that asymmetry in inter-cusp spacing results in profiles that are skewed towards the region of lower-B field. Interestingly, in Case 3 the left-most cusp appears to be collecting almost zero current, which may be a result of both cusp placement asymmetry and filament location.

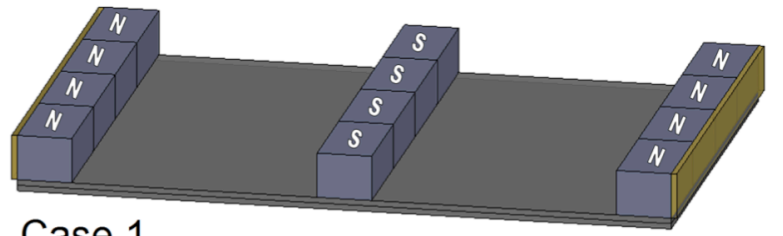

Case 1

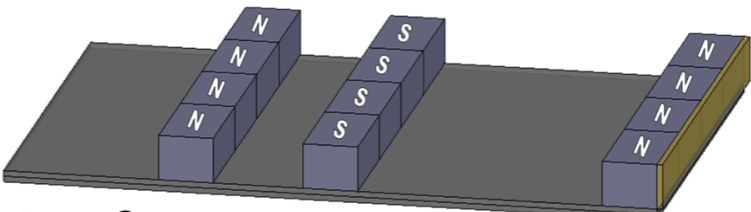

Case 2

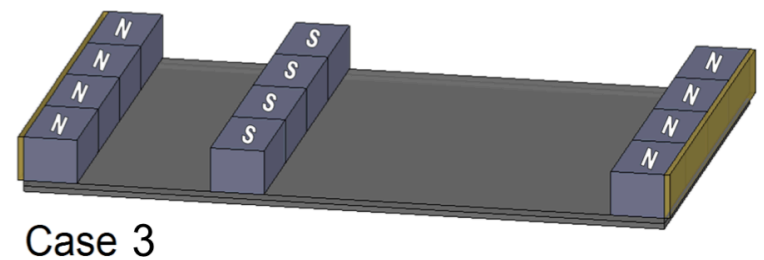

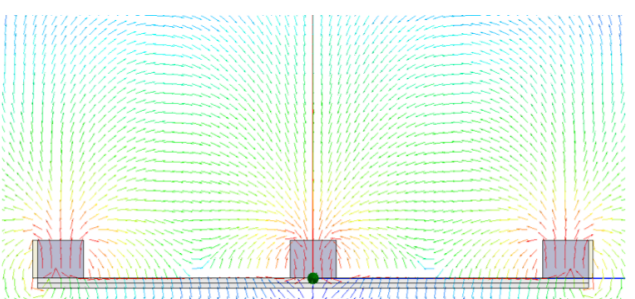
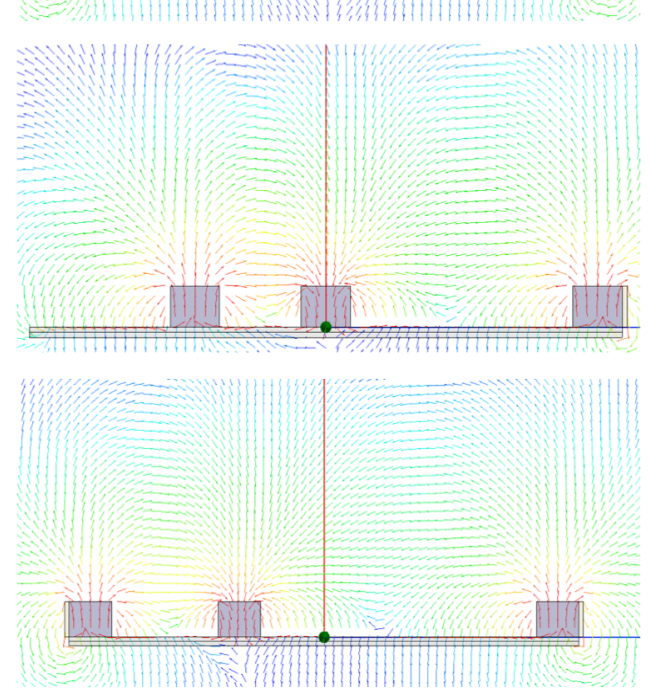

Figure 10: (Left) Anode configurations used for the study of inter-cusp spacing on cusp collection. (Right) Magnetic field vector plots for each configuration. 

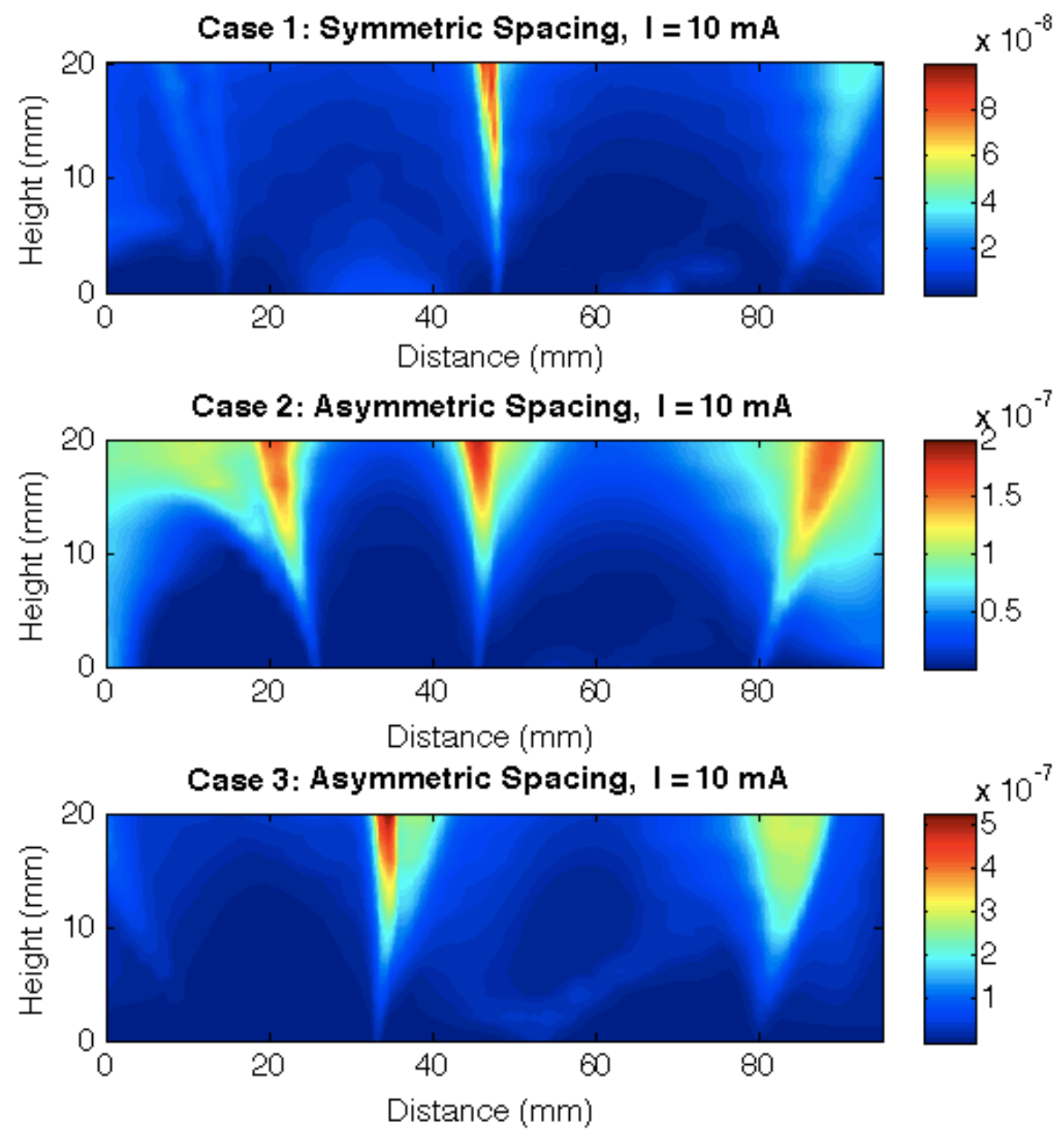

Figure 11: Raw probe current (A) as a function of position relative to the anode plate. Cases correspond with the anode configurations depicted in Figure 10. Note the difference in scale between the three plots.

Cusp profile width and transmission were evaluated over the central cusp for all three cases. Profile FWHM vs. probe height was plotted against twice the primary electron Larmor radius for each configuration in Figure 12. Transmission was determined by the same method as described in section 3.A.1, and is plotted for each configuration in Figure 13. As discussed earlier, the current profile shapes represent the raw acquisition data and have not been corrected for line broadening due to finite size of the probe. Near the anode surface there is no difference in loss width within the uncertainty in the measurement; however above $1 \mathrm{~cm}$ the profiles in the asymmetric cases are somewhat wider than in the symmetric case. In addition, asymmetry appears to reduce transmission from about $20 \%$ in the symmetric case to about $5 \%$. This can be seen quite clearly in the raw probe current shown in Figure 11. Although discharge current remains constant at $10 \mathrm{~mA}$ between the three cases, there are clearly many more electrons present in the cusp region in the asymmetric cases. Based on these limited tests, cusp symmetry appears to play a stronger role on electron collection that simple filament placement; that is, local magnetic structure appears to be more important than electron emission source location. 


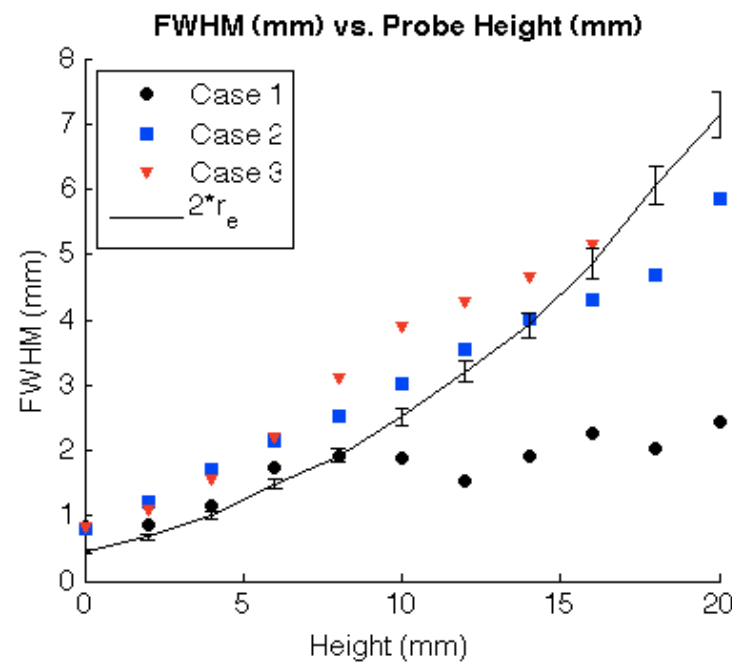

Figure 12: FWHM (mm) as a function of probe height above the magnet surface $(\mathrm{mm})$. These widths are compared with twice the primary electron Larmor radius (mm).
Transmission vs. Probe Height $(\mathrm{mm})$

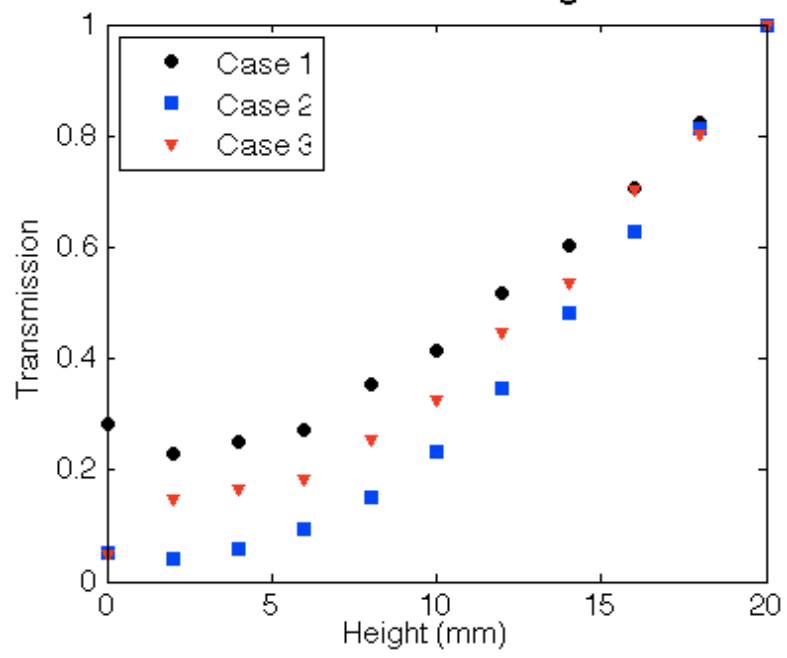

Figure 13: Transmission as a function of probe height above the magnet surface $(\mathrm{mm})$.

\section{B. 20-cm Conic Discharge Chamber}

Three ionization probabilities were investigated for this study: the case of no gas flow, which corresponds to an ionization probability of about $1.4 \%$, and two levels of argon gas flow corresponding to ionization probabilities of $20 \%$ and $40 \%$. At each flow rate, a high and low discharge current case was studied by adjusting the current applied to the filament. Six sets of data were thus obtained. As described above and depicted in Figure 5, probe current was measured along a plane perpendicular to the magnet surface over two of the magnet rings. In addition, bulk probe I$\mathrm{V}$ traces were taken for the data sets taken at $20 \%$ and $40 \%$ ionization probability. These are shown in Figure 14.

As flow and discharge current increases, electron and ion current increases in the bulk region, as expected. At low flow rates there is almost no detectable ion current (within the limitations of the measurement apparatus), as expected when the rate of ionization is low. At low flow rates we thus expect the majority of electron current measured to be due to primary electrons. As flow increases, more and more current is expected to be due to plasma electrons. This is expected to increase the loss width towards the hybrid radius as opposed to the primary Larmor radius.

Raw cusp probe current is plotted as a function of position relative to the anode for the six cases investigated in Figure 15. In all cases it is obvious that the majority of discharge current is collected at the first of the two magnet cusps investigated. This is the cusp that is closest to the filament; electrons emitted from the filament appear to be transported to the cusp region of this nearest magnet ring. The cusp region is taken to be the region between the magnet surface and a probe height of $2 \mathrm{~cm}$; above this region we take to be the bulk discharge. Analysis of these regions is split into two parts: an analysis of profile width and an analysis of transmission as a function of

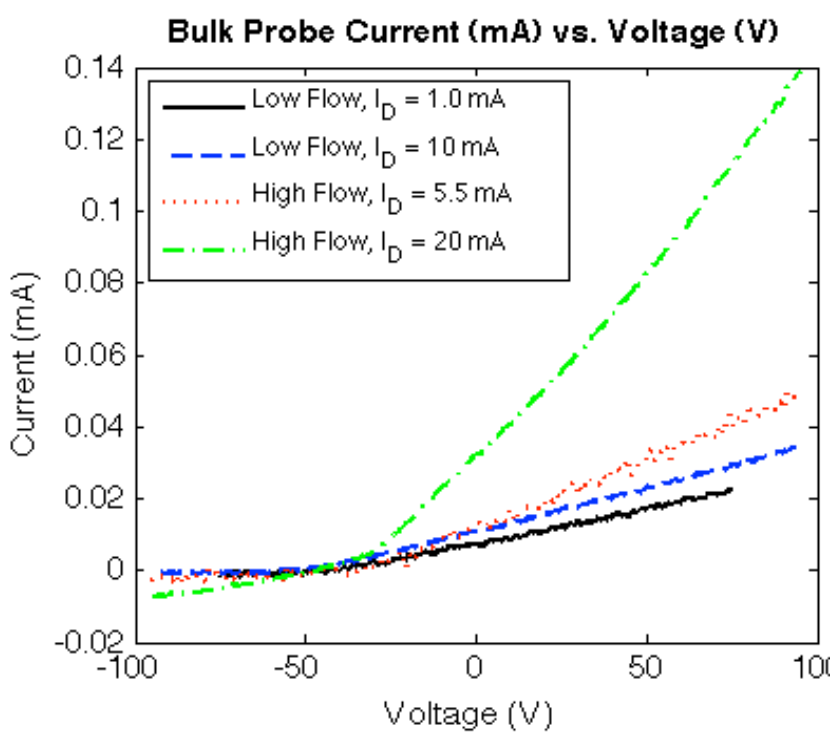

Figure 14: Bulk (field-free) IV traces taken at various pressures and discharge currents.

8

American Institute of Aeronautics and Astronautics 

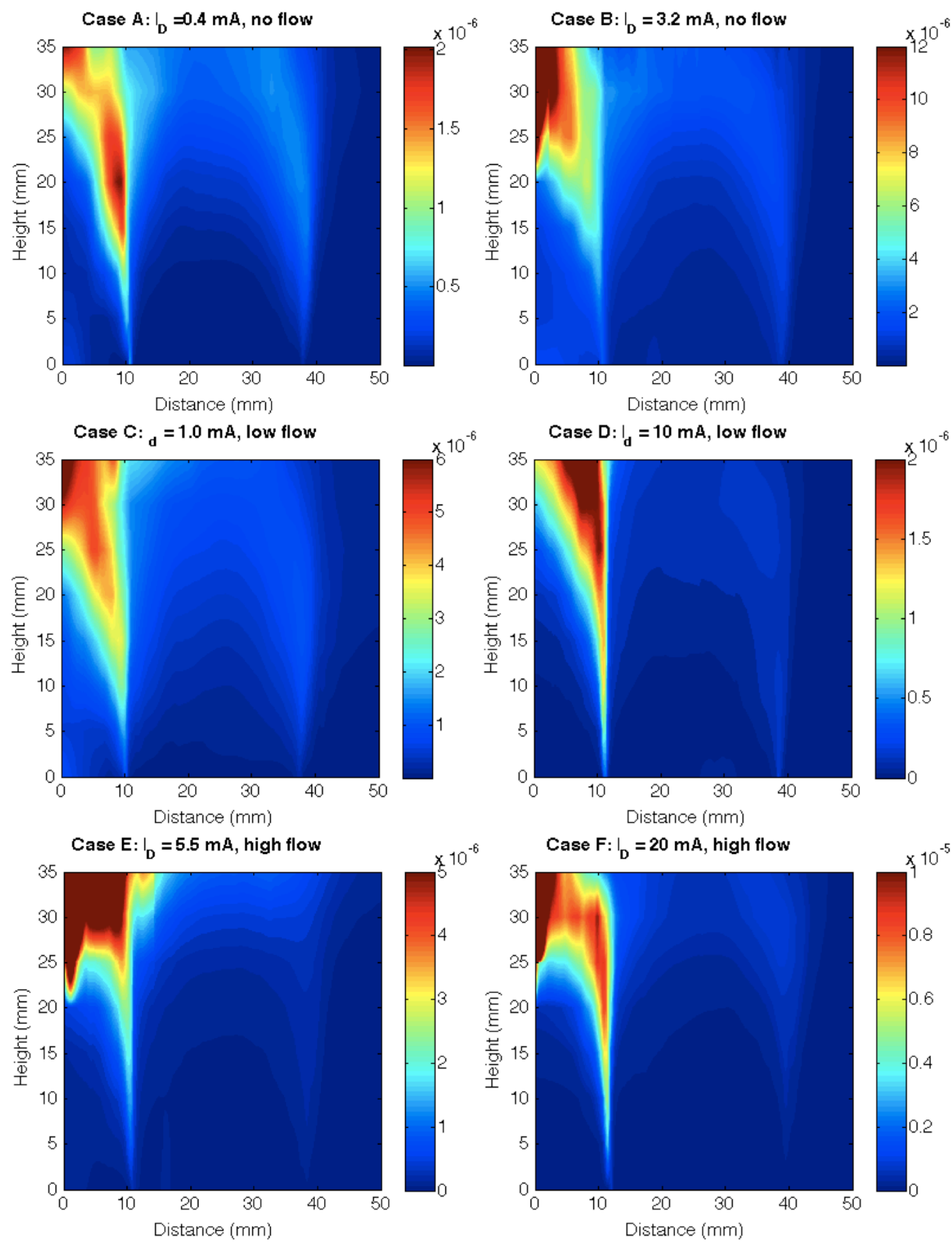

Figure 15: Raw probe current plotted as a function of position relative to the anode. A high and low discharge current case was investigated at each pressure studied. Low flow corresponds to an ionization probability of about $20 \%$, and high flow corresponds to an ionization probability of about $40 \%$. 


\section{Profile Width}

The FWHM of the two cusp profiles was determined in each of the six cases studied to assess sensitivity to discharge current and gas flow. Profile widths are compared to twice the primary electron Larmor radius as well as four times the hybrid radius (the leak width as given by Hershkowitz ${ }^{18}$ ). FWHM is plotted as a function of probe height in Figure 16 for both magnet rings investigated as well as both high and low discharge current cases. Ring 2 is the closer of the two rings studied to the filament, at which most of the current appeared to be collected. Profile widths over ring 2 are seen in figures 16a and 16c. and widths over ring 3 are seen in figures 16b and 16d. Widths are compared to twice the primary electron Larmor radius $(\mathrm{mm})$, the primary electron leak width, as well as four times the hybrid radius $(\mathrm{mm})$, the hybrid loss width. The hybrid radius was calculated by assuming a plasma electron temperature of $10 \mathrm{eV}^{4}$ and an ion temperature of $0.1 \mathrm{eV}$.

Generally widths appear to hover between twice the primary electron Larmor radius and the hybrid loss width. Oddly, at the highest flow rate widths appear to be narrowest, which ran contrary to the expectation that as ionization increases the loss width should tend towards the hybrid loss width. In the case of no gas flow, leak width increased with discharge current, however this was not always the case at increased levels of ionization. Despite the much higher current collected at the second ring relative to the third, there does not appear to be a significant difference in leak width between the two rings studied. At low levels of ionization, leak width (as measured by profile FWHM) is between the primary electron and hybrid leak widths. It is important to note that widths

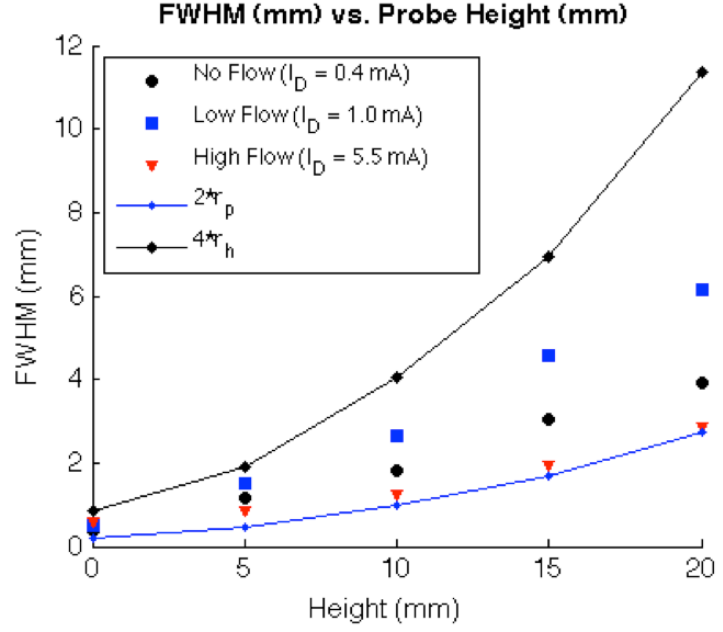

16a: Ring 2, Low $I_{D}$

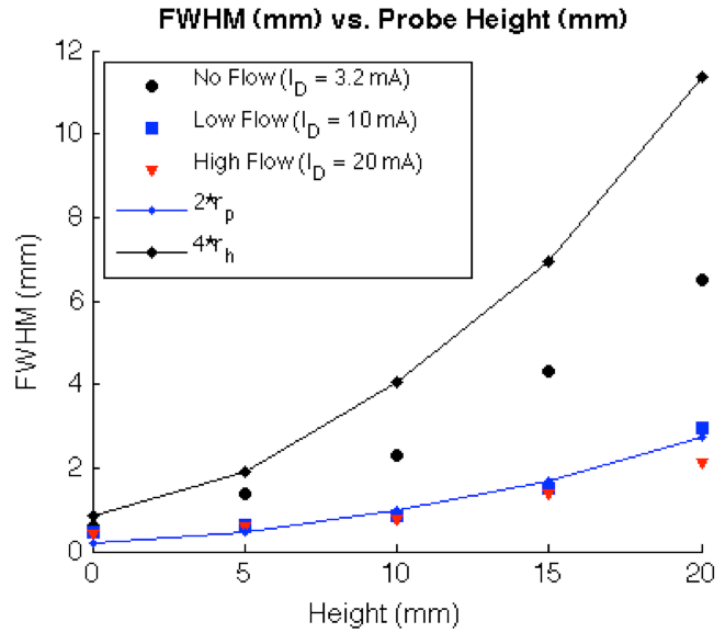

16c: Ring 2, High $I_{D}$

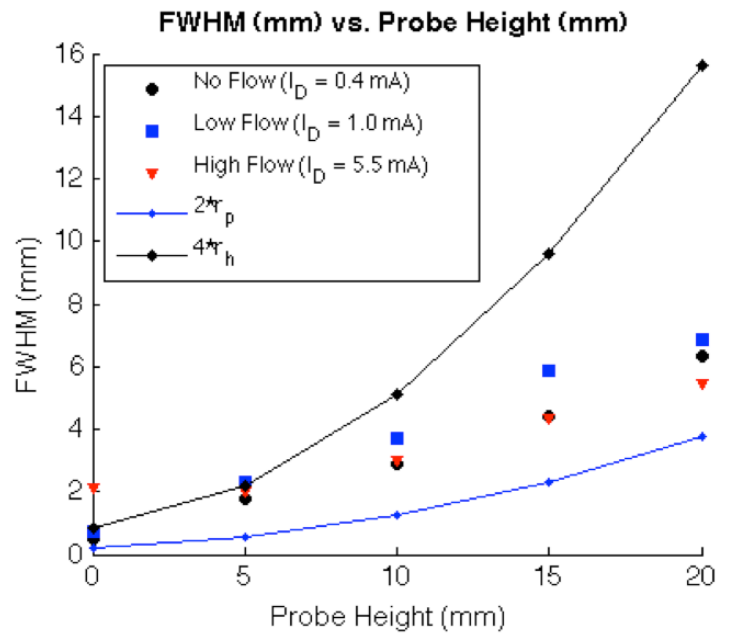

16b: Ring 3, Low $I_{D}$

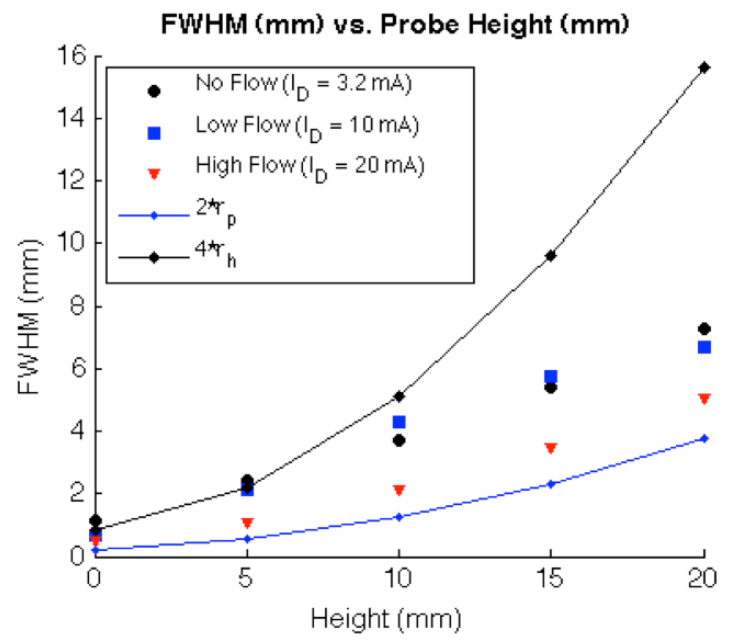

16d: Ring 3, High I

Figure 16a-d: FWHM (mm) as a function of probe height compared over three different flow rates. 

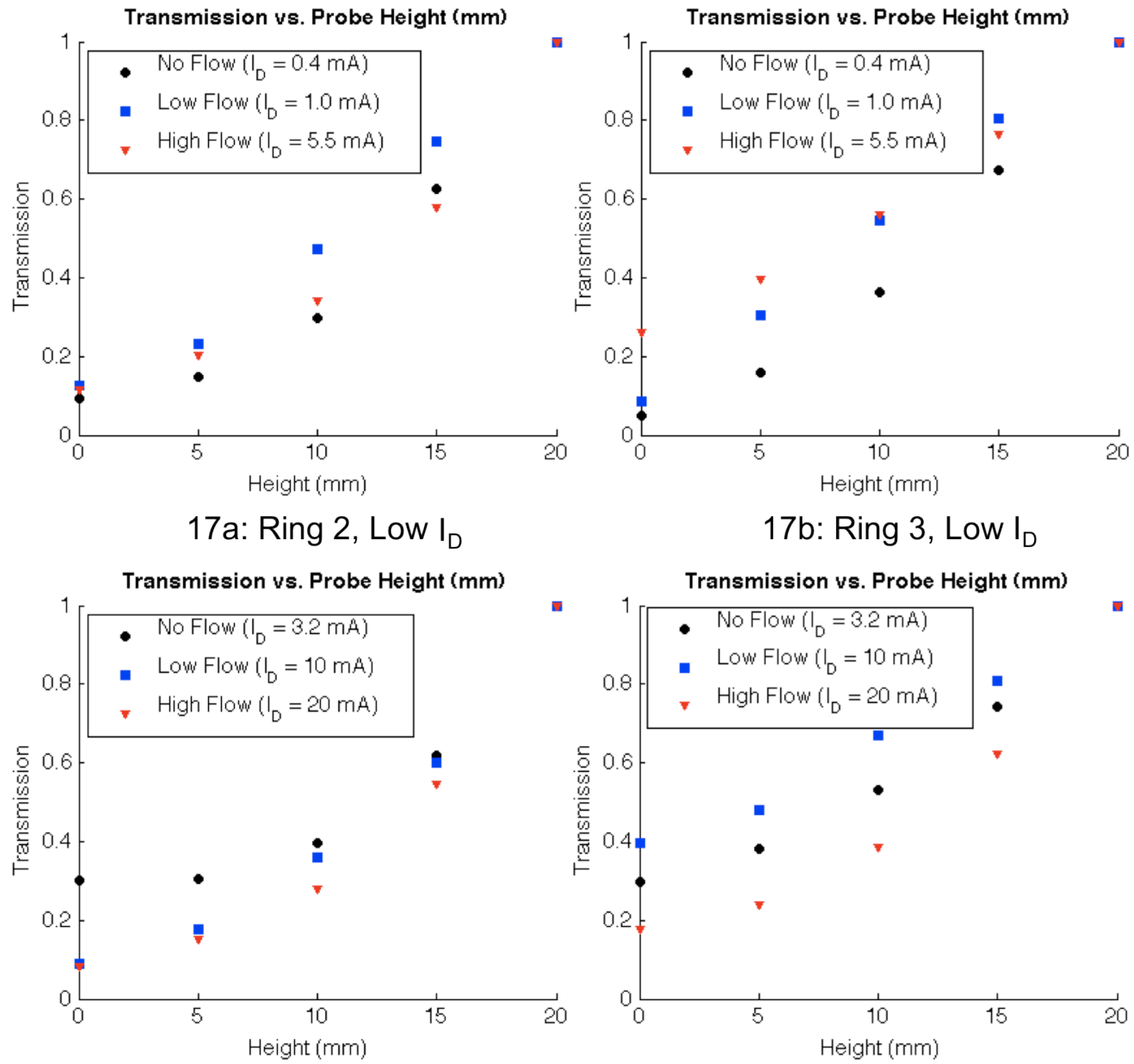

17c: Ring 2, High $I_{D}$

17d: Ring 3, High $I_{D}$

Figure 17: Transmission as a function fo probe height compared over three different flow rates.

calculated from asymmetric, non-Gaussian profiles may be inaccurate and the confusing trends seen in this data may be the result of artifacts from extracting data from these profiles. All that can be definitively drawn from this data is that loss widths fall between the primary loss width and the hybrid loss width.

\section{Transmission}

Transmission was determined by the same method as described in 3.A.1, and plotted in Figure 17. Considering first only the case of zero gas flow, we see a transmission coefficient of about 5-10\% in the case of low discharge current. At high discharge current, the transmission increases to about $30 \%$. In the two cases of gas flow there appears to be some variation in transmission, with values ranging from about $10 \%$ to nearly $40 \%$. Transmission appears to be largely independent of discharge current in the case of ring 2, but in ring 3 there are marked differences in transmission depending on discharge current for a given flow rate (with the exception of high flow rate, for which transmission remains fairly steady around 20\%). It does not seem that a consistent formula for determining transmission as a function of discharge current and ionization probability can be determined from this 
data; many more data sets and varying conditions will be required, and additional data sets at these same conditions will also be required to determine repeatability of these measurements.

\section{Conclusion}

This series of experiments expanded upon former work in an attempt to quantify primary electron losses at the anode in a multipole cusped source. Two anode configurations were studied in this work, a planar line cusp source and a $20-\mathrm{cm}$ conic discharge chamber. A translatable Langmuir probe was used to measure 2D probe current density maps in the cusp region, allowing for the visualization of electron transport through the cusps. Profile width and transmission could then be calculated as a function of operating parameters for each source.

In the case of the planar line cusp source, it was found that the uncorrected profile width corresponded closely to twice the primary Larmor radius. Profile corrections are expected to reduce the width of the measured profiles. In the case of a symmetric configuration transmission was measured to be around $20 \%$; when asymmetry in the cusp spacing was introduced transmission was cut to only 5\%. This has implications for magnetic circuit design, since decreased transmission is favorable in reducing losses to the cusp.

Transmission and leak width was measured in the conic discharge chamber as a function of both discharge current and gas flow rate. In all cases the leak width was between the primary electron leak width $\left(2 * r_{p}\right)$ and the hybrid leak width $\left(4 *_{h}\right)$. A better method of extracting widths from the measured current profiles is required to more accurately quantify electron losses. In the case of no gas flow transmission ranged from about $10 \%$ at low discharge current to $30 \%$ at high discharge current.

\section{Acknowledgments}

A.A. Hubble thanks the Department of Defense (DoD) for support through the National Defense Science \& Engineering Graduate Fellowship (NDSEG) Program.

\section{References}

${ }^{1}$ R.A. Bosch, R.L. Merlino, Phys Fluids 29, 1998 (1986).

${ }^{2}$ C. Koch, M. Matthieussent, Phys. Fluids 26, 545 (1982).

${ }^{3}$ H. Horiike, A. Masato, O. Yoshihiro, Y. Okumura, S. Tanaka, Phys Fluids 30, 3268 (1987).

${ }^{4}$ G. Knorr, R.L. Merlino, Plasma Phys Contr F 26, 443 (1984).

${ }^{5}$ R.E. Pechacek et al, Phys Rev Lett 45, 256 (1980).

${ }^{6}$ S.W. Hwang, Y.J. Lee, H.R. Han, J.B. Yoo, G.Y. Yeom, J Vac Sci Technol A 17, 1211 (199).

${ }^{7}$ T. Morishita, M. Ogasawara, A. Hatayama, Rev Sci Instrum 69, 968 (1998).

${ }^{8}$ D. Goebel, Phys Fluids 25, 1093 (1982).

${ }^{9}$ A. Anukaliani, V. Selvarajan,, Eur Phys J AP 15, 199 (2001).

${ }^{10}$ Y. Arakawa, C. Hamatani, Proceedings of the $19^{\text {th }}$ International Electric Populsion Conference, Colorado Springs, AIAA Paper 87-1079 (1987).

${ }^{11}$ R. Limpaecher, K.R. MacKenzie, Rev Sci Instrum 44, 726 (1973).

${ }^{12}$ C. Gauthereau, G. Matthieussent, Phys Lett 102A, 231 (1984).

${ }^{13}$ T. Lagarde, J. Pelletier, Y. Arnal, Plasma Sources Sci Technol 6, 53 (1997).

${ }^{14}$ G.M. Sandontano, J.J. Barroso, A. Montes, IEEE T Plasma Sci 24, 1319 (1996).

${ }^{15}$ R. Wirz, D. Goebel, Proceedings of the $42{ }^{\text {nd }}$ AIAA/ASM/SAE/ASEE Joint Propulsion Conference, AIAA Paper 2006-4487 (2006).

${ }^{16}$ J.M. Buzzi, J. Snow, J.L. Hirshfield, Phys Lett 54A, 344 (1975).

${ }^{17}$ H. Kozima, S. Kawamoto, K. Tamagiwa, Phys Rev Lett 86A, 373 (1981).

${ }^{18}$ N. Hershkowitz, K.N. Leung, Romesser, Phys Rev Lett 35, 227 (1975).

${ }^{19}$ R. Jones, Plasma Physics 21, 505 (1979).

${ }^{20}$ Y. Arakawa, C. Hamatani, J Propul Power 3, 90 (1979).

${ }^{21}$ J.S. Sovey, J Spacecraft 21, 488 (1983).

${ }^{22}$ K.N. Leung, T.K. Samec, A. Lamm, Phys Lett 51A, 490 (1975).

${ }^{23}$ M. Sadowski, Phys Lett 28A, 626 (1969).

${ }^{24}$ A. Sengupta, J. Appl. Phys. 105, 093303 (2009).

${ }^{25}$ A.A. Hubble, J.E. Foster, Proceedings of the $44^{\text {th }}$ AIAA/ASM/SAE/ASEE Joint Propulsion Conference, Hartford, AIAA Paper 2008-4639 (2008).

${ }^{26}$ A.A. Hubble, J.E. Foster, Proceedings of the $31^{\text {st }}$ International Electric Propulsion Conference, Ann Arbor, IEPC-2009-156 (2009).

${ }^{27}$ D. Goebel, R. Wirz, I. Katz, J Propul Power 23, 1055 (2007).

${ }^{28}$ M. Lieberman, A. Lichtenberg, Principles of Plasma Discharges and Materials Processing, $2^{\text {nd }}$ ed., 2005. 